\title{
Incidência de hanseníase na Paraíba entre os anos de 2010 a 2014
}

\section{Incidence of leprosy in Paraíba between the years 2010 to 2014}

\author{
Sylmara Patricio de Santana Rosa \\ Graduada em Farmácia pela FASP - Cajazeiras - PB E-mail silmara_patricia13@hotmail.com \\ Vivianne Macelino de Medeiros \\ Doutorado em Produtos Naturais e Sintéticos Bioativos-UFPB \\ Samara Raquel Souza Ribeiro Andrade \\ Graduanda em Química, IFPB-SOUSA/PB. E-mail:samararibeiroa@gmail.com \\ Orestes Quércias de Medeiros \\ Graduado em Enfermagem-Faculdade de Ciências Médicas-João Pessoa-PB-E-mail:orestesquercias@hotmail.com \\ Aline Carla de Medeiros \\ Doutoranda em Engenharia de Processos-PPGEP-UFCG-Campina Grande-PB
}

\begin{abstract}
Resumo: A hanseníase é uma doença causada pelo Mycobacterium leprae. Caracteriza-se por atingir predominantemente a pele e os nervos periféricos. Seu diagnóstico ainda causa grande impacto psicossocial e comprometimento da qualidade de vida. Apresenta-se como uma das doenças mais antigas da humanidade e apesar da cura existir, ainda constitui importante problema de saúde pública no Brasil. No estudo objetivou-se analisar a incidência dos casos de hanseníase notificados no estado da Paraíba no período de 2010 à 2014. Para a realização do estudo foram analisados os casos notificados e confirmados de hanseníase pelo Sistema de Informação de Agravos de Notificação (SINAN) durante o período de estudo. Foram registrados 3727 casos durante os anos de 2010 a 2014 , analisando-os observou-se uma maior predominância da doença nos pacientes do sexo masculino e de acordo com a classificação operacional houve prevalência multibacilar. Além disso, a forma clínica Dimorfa, foi a forma mais comum. De acordo com o tipo de saída, a maior parte dos pacientes evoluiu pra cura, fato justificado pela eficiência da poliquimioterapia associada ao acompanhamento multiprofissional na prevenção e tratamento de incapacidades. Com a realização desta pesquisa pode-se constatar que existem diferenças significativas no perfil da hanseníase ao longo dos anos analisados. De forma geral, os índices da doença vêm diminuindo, porém de forma lenta. Sugere-se campanhas educativas, para que a população quebre o preconceito e se conscientize procurando o mais rápido possível o atendimento médico, buscando a cura e evitando a disseminação da doença.
\end{abstract}

Palavras-chave: Hanseníase; Multibacilar; Saúde Coletiva

\begin{abstract}
Leprosy is a disease caused by Mycobacterium leprae. It characterized by affecting predominantly the skin and peripheral nerves. Diagnosis still a major psychosocial impact and impaired quality of life. It is presented as one of the oldest diseases of mankind and despite the cure exists, is still a major public health problem in Brazil. The study aimed to analyze the incidence of leprosy cases reported in the state of Paraiba in the period 2010 to 2014 . To carry out such study, reported and confirmed cases of leprosy by the Notifiable Diseases Information System were analyzed (SINAN) during the study period. 3727 cases were reported during the period from 2010 to 2014, analyzing them there was a greater prevalence of the disease in male patients and according to operational classification was prevalence multibacillary. Furthermore, the clinical form Dimorph, was the most common form. According to the type of output, most of the patients progressed to cure, which may be explained by the efficiency associated with multidrug multi monitoring the prevention and treatment of disabilities. With this research can be seen that there are significant differences in the leprosy profile over the years analyzed. In general, rates of the disease have been declining, however slowly. It is suggested educational campaigns for the population to break the prejudice and become aware looking for the fastest possible medical care, seeking healing and preventing the spread of disease.
\end{abstract}

Keywords: Leprosy; Multibacillary; Collective Health 


\section{INTRODUÇÃO}

A hanseníase é uma doença causada pelo Mycobacterium leprae. Caracteriza-se por atingir predominantemente a pele e os nervos periféricos. Seu diagnóstico ainda causa grande impacto psicossocial e comprometimento da qualidade de vida. Apresenta-se como uma das doenças mais antigas da humanidade e apesar da cura existir, ainda constitui importante problema de saúde publica no Brasil, sendo considerada uma doença endêmica, fato que torna o país responsável por $93 \%$ dos casos de hanseníase no continente americano (MELÃO et al., 2011; SOUSA et al., 2013). No ano de 2007 o coeficiente de detecção da hanseníase no Brasil foi de 21,08/100.000 habitantes e o coeficiente de prevalência, de 2,19/10.000 habitantes (LIMA et al., 2010).

A hanseníase está presente nas diferentes regiões do país, e afeta indivíduos independentemente de faixa etária, raça e até mesmo nível socioeconômico. Quando não tratada, deixa grave sequelas e/ou mutilações físicas e sociais (XIMENES NETO et al., 2010).

A doença é dividida em quatro formas: Indeterminada, tuberculoide, dimorfa e virchowiana. A forma de manifestação da infecção da doença pode evoluir das diferentes formas, de acordo com a resposta imunológica do hospedeiro para com o bacilo. $\mathrm{O}$ diagnóstico baseia-se em sinais cardinais, como lesões cutâneas com falta de sensibilidade, espessamento de nervos periféricos, a baciloscopia é o exame complementar mais confirmativo. $\mathrm{O}$ tratamento da hanseníase compreende: quimioterapia específica, supressão dos surtos reacionais, prevenção de incapacidades físicas, reabilitação física e psicossocial (ARAÚJO, 2003).

Mesmo com as políticas e ações estratégicasdiagnóstica com tratamento e cura efetiva, a taxa de detecção de novos casos permanece alta em diferentes regiões do mundo. De acordo com a Organização Mundial da Saúde (OMS), o Brasil apresentou coeficiente de 2,10 casos de hanseníase por 10.000 habitantes em 2007 (XIMENES NETO et al., 2010; SOUSA, 2012). Sendo as regiões Nordeste, Norte e Centro-oeste as que possuem um maior número de notificações de casos no país (MAGALHÃES; ROJAS, 2007).
De acordo com o SINAN, em 2010 foram evidenciados 912 casos de hanseníase notificados no estado da Paraíba, foi realizado uma análise nos estados que mais notificaram casos, sendo 15 municípios onde foi possível observar maior incidência da doença nas cidades de Campina Grande, João Pessoa e Cajazeiras (SIMPSON et al., 2010).

Nesse sentido objetivou-se analisar a incidência dos casos de hanseníase notificados no estado da Paraíba no período de 2010 à 2014.

\section{MATERIAIS E MÉTODOS}

Trata-se de um estudo transversal retrospectivo em forma de uma pesquisa descritiva, onde os fatos foram registrados, analisados, classificados e interpretados sem a interferência do pesquisador.

Foram obtidos dados sobre á incidência de casos de hanseníase no estado da Paraíba, no período de 2010 á 2014. Os dados foram obtidos através do SINAN (Sistema de Informação de Agravos de Notificação), o qual realiza notificações e investiga doenças e agravos que constam na lista nacional de doenças de notificação compulsória incluindo á hanseníase.

Os dados coletados foram analisados e expressos na forma de tabelas e gráficos construídos no programa Microsoft Office Excel, versão 2010, e posteriormente discutidos com base na literatura pertinente a temática para melhorar apresentação das variáveis.

\section{RESULTADOS E DISCUSSÃO}

As informações foram coletadas no banco de dados do Sistema de informação e Agravos de Notificação (SINAN). Após analisados, os dados foram apresentados em tabelas e gráficos de acordo com as variáveis: sexo, raça, classificação operacional, forma clínica e tipo de saída. Todos os casos foram notificados somente no estado da Paraíba no período de 2010 a 2014.

Os resultados revelaram 3727 casos notificados de hanseníase no estado da Paraíba, no período de 2010 a 2014. Sendo o ano de 2011 o período com o maior número de registro de casos e o ano de 2014 o período com menores notificações de casos (Figura 01)

Figura 01. Número de casos de Hanseníase notificados no estado da Paraíba, no período de 2010 a 2014.

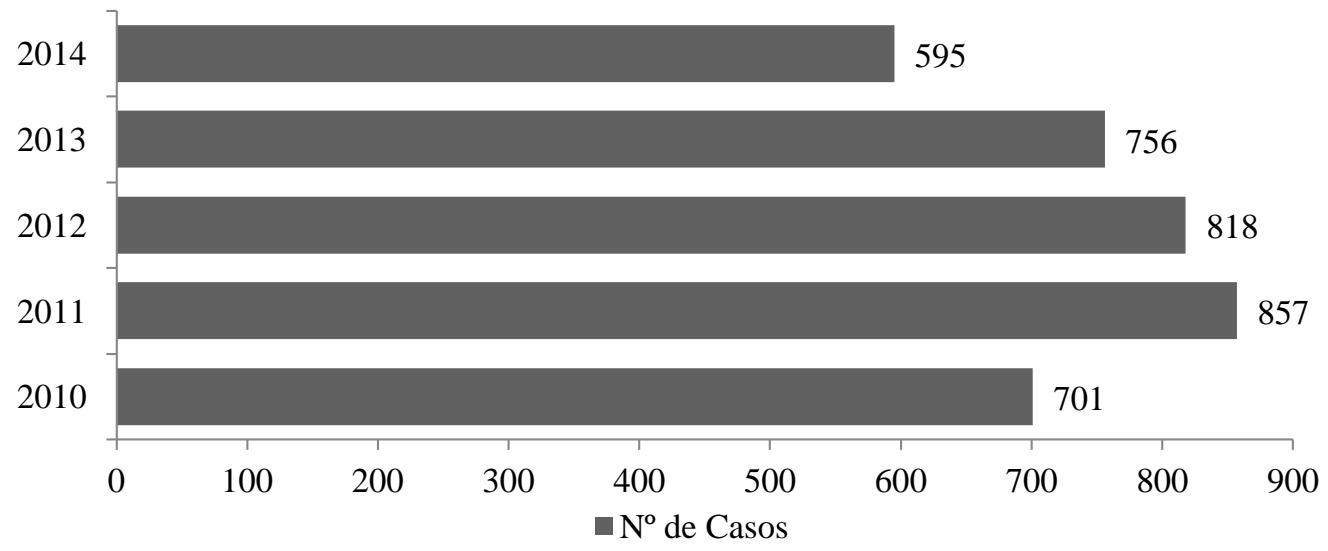


Tabela 01- Número de casos de Hanseníase notificados no estado da Paraíba de acordo com a variável raça, no período de 2010 a 2014.

\begin{tabular}{|c|c|c|c|c|c|}
\hline Raça & Ano 2010 & Ano 2011 & Ano 2012 & Ano 2013 & Ano 2014 \\
\hline Branco & 214 & 228 & 237 & 206 & 173 \\
\hline Preta & 85 & 107 & 95 & 75 & 74 \\
\hline Amarela & 3 & 15 & 19 & 9 & 5 \\
\hline Parda & 371 & 473 & 423 & 433 & 308 \\
\hline Indígena & 3 & 7 & 4 & 1 & 1 \\
\hline Ignorado/Branco & 20 & 28 & 40 & 32 & 31 \\
\hline
\end{tabular}

Analisando a tabela 01 , notamos uma elevada predominância de casos na cor parda, fato relacionado à região onde foi realizado o estudo. Na região do Nordeste onde se localiza o estado da Paraíba existe um maior número da população parda, sendo assim os percentuais apresentam-se mais elevados, diferente do estado Sul do Brasil que provavelmente se destacariam as raças brancas (BRITO et al., 2014). O menor índice da doença foi na raça indígena, até porque o perfil dos povos indígenas é muito pouco conhecido, ocasionando ausência de investigação. Não se pode considerar o pequeno número de casos nesta raça, como baixa endemia da doença, visto que o acesso á comunidades indígenas ainda é muito pequeno (IMBIRIBA et al., 2009). Houve um expresso número ignorado ou não preenchido, o que representa a dificuldade que o SINAN enfrenta por falta de atenção dos profissionais que preenchem a ficha de notificação.

Com relação ao gênero, os resultados revelam uma significativa prevalência da doença no sexo masculino. Com um número de casos bem próximo de ambos os sexo no ano de 2012 (Figura 02).

Figura 02. Número de casos de Hanseníase notificados no período de 2010 a 2014 de acordo com o gênero do paciente.

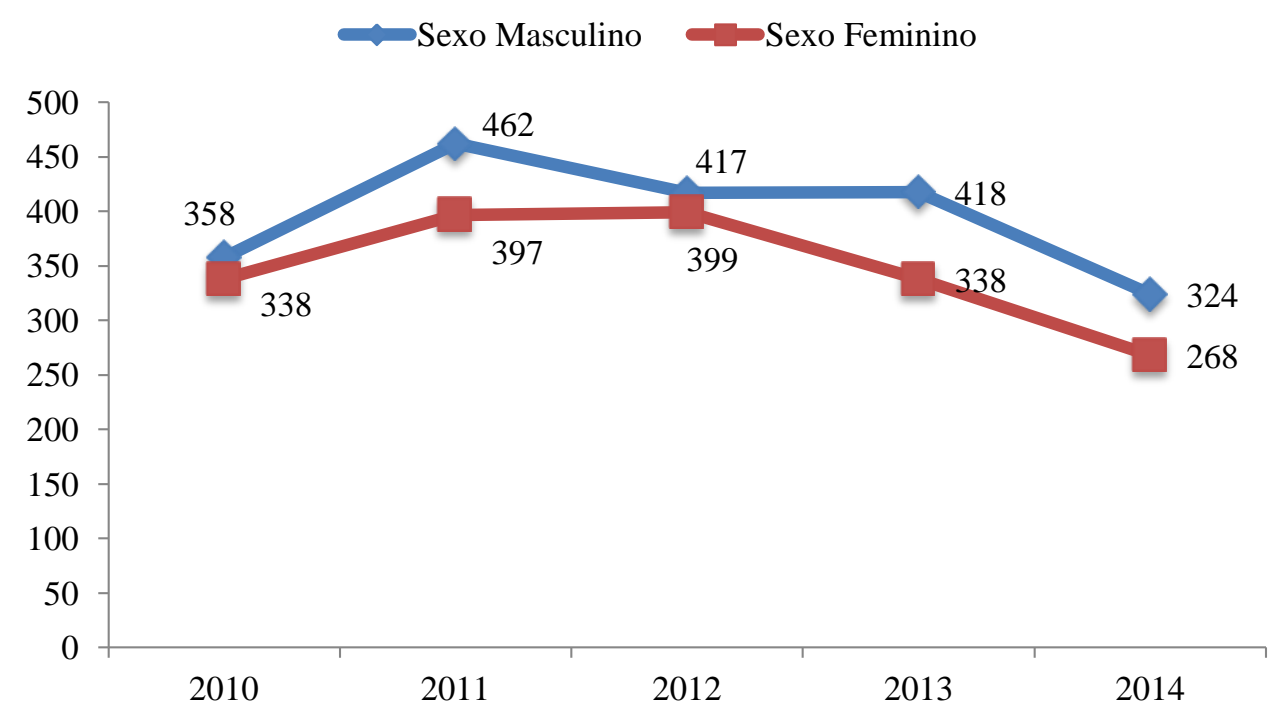

É possível notar o domínio do sexo masculino acometido pela hanseníase, o que pode ser explicado devido às condições de vida mais ativas dos homens, estando assim mais expostos á doença (SIMPSON et al., 2010). No ano de 2012 com mais oportunidades de emprego para o sexo feminino e por tanto, aumento da inclusão das mulheres no mercado de trabalho, houve um aumento no número de notificações de casos na Paraíba. Além de uma maior exposição, as mulheres costumam procurar mais que os homens o atendimento médico.
Muitos estudos comprovam que os homens evitam a busca do serviço de saúde mais do que as mulheres e também morrem mais do que elas. Isso está associado as práticas masculinas e a defesa de aspectos culturais (GOMES et al., 2007).

As formas clinicas predominante da doença, foram as formas dimorfa e tuberculoide. Sendo a forma dimorfa de grande poder de transmissão a mais prevalente (Figura 03). 
Figura 03. Números de casos de Hanseníase notificados de acordo com a forma clínica da doença, no período de 2010 a 2014.

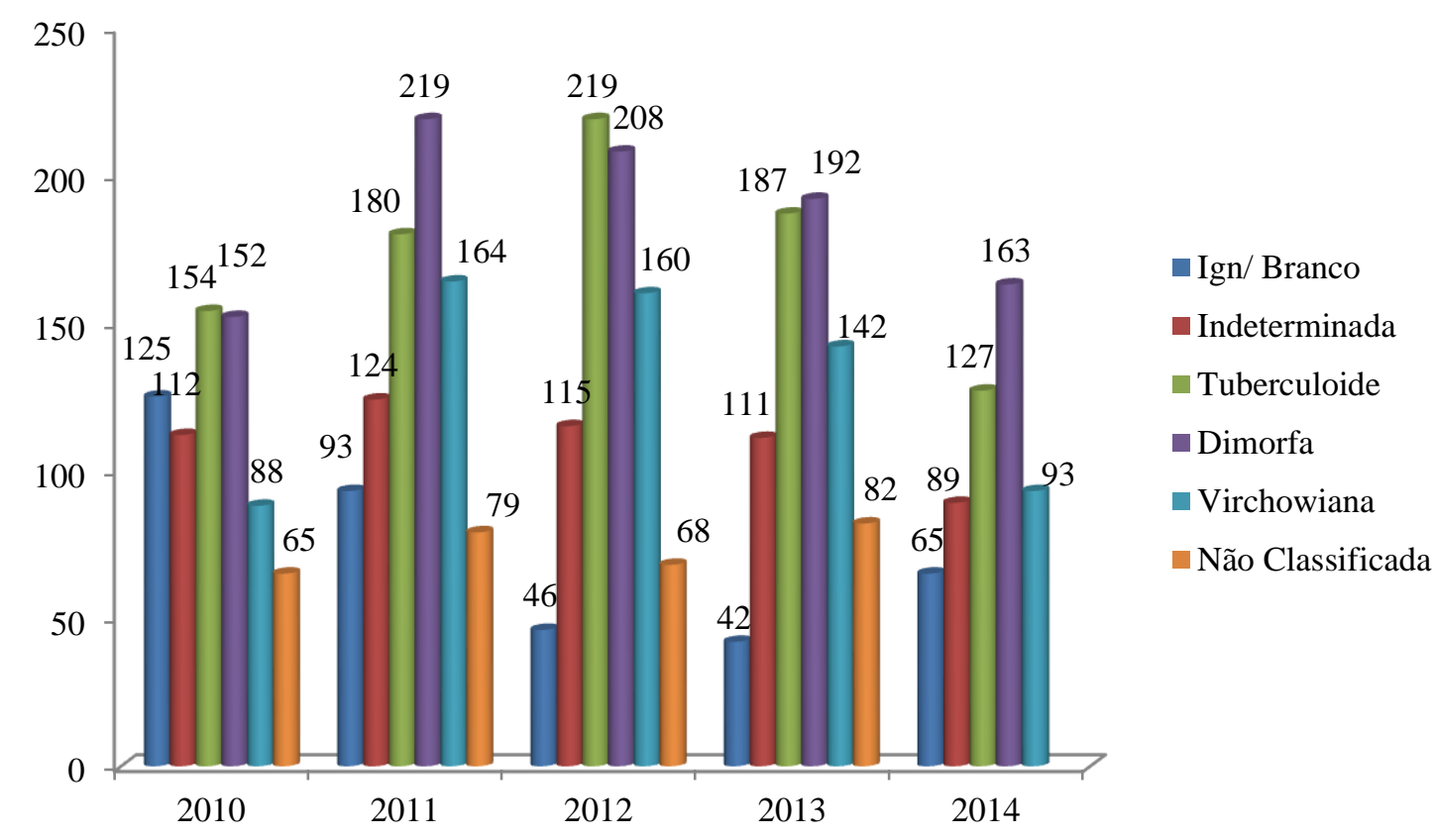

A forma clínica predominante mais acometida foi à hanseníase Dimorfa, essa forma é considerada a mais instável caracterizada pelo comprometimento neurológico troncular e episódios reacionais, aumentando as chances dos pacientes desenvolverem incapacidades e deformidades físicas (SOUZA, 1997). Essa forma foi a de maior prevalência por uma falha no diagnóstico ou diagnóstico tardio da doença. Geralmente os doentes acometidos só procuram a unidade de saúde quando a doença já está em estado bastante elevado. Este é um dado preocupante, por ser uma das formas clinica mais desenvolvida da doença $\mathrm{E}$ em segundo lugar a forma Tuberculoide acometeu os doentes, sendo esta a forma benigna da doença, geralmente apresenta-se na forma de lesões únicas ou em pequenos números, indica que a doença foi diagnosticada no inicio de seu aparecimento.

Ao observar a Figura 04 percebe-se a predominância da hanseníase na forma multibacilar que constituem o grupo contagiante da doença.

Figura 04. Frequência dos casos de hanseníase de acordo com a classificação operacional. No período de 2010 à 2014.

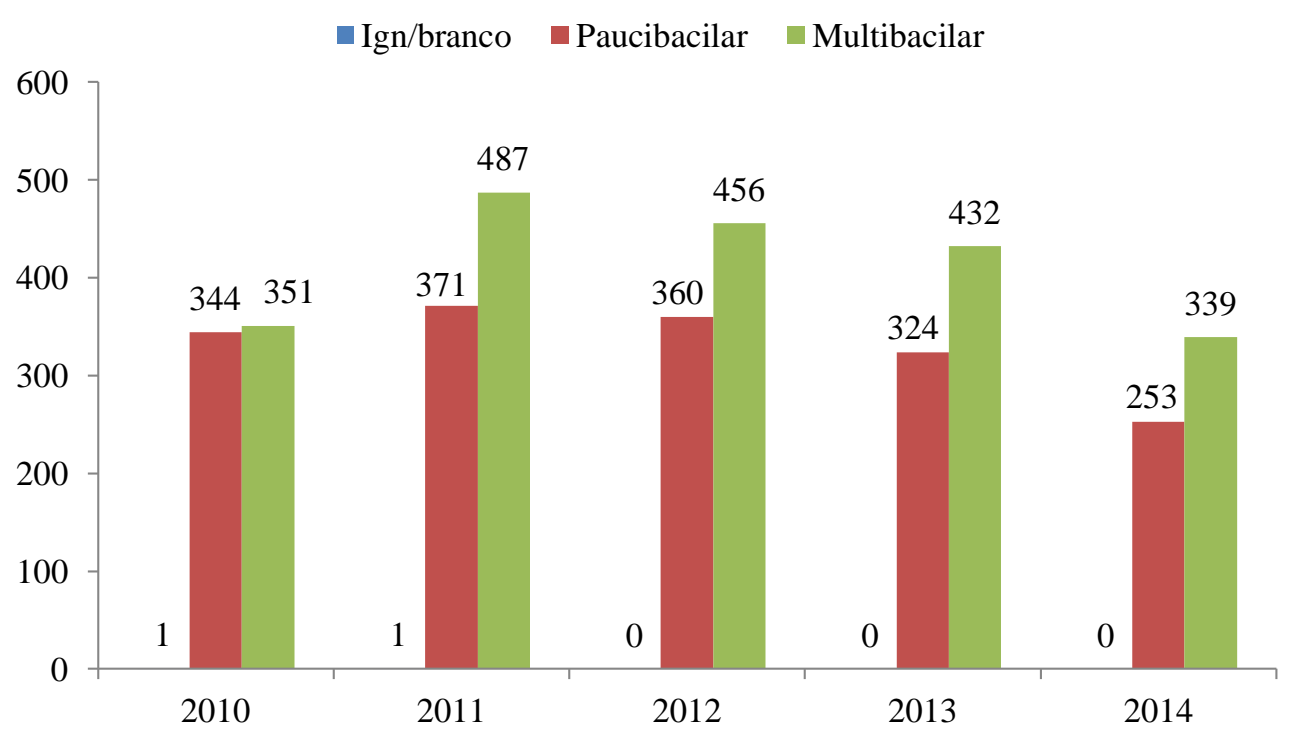

O dado da classificação operacional da doença é de extrema importância para determinar o tratamento quanto ao tipo e o tempo do esquema quimioterápico, foi observada a prevalência da forma multibacilar que gera preocupação por sua forma de acometimento e grau de disseminação. A hanseníase pode ser comumente categorizada utilizando-se a nomenclatura de Madri, ou a classificação simplificada da OMS. Considera-se paucibacilares casos com até 5 lesões e diagnosticados clinicamente nas formas indeterminada ou tuberculóide, e multibacilares os casos com mais de cinco lesões e classificadas nas formas dimorfa e Virchoviana (BRITO et 
al, 2014). A prevalência de doentes enfestados de lesões na forma multibacilar indica um risco de contágio maior, por ser a forma que os bacilos são encontrados em maiores quantidade e possuem maior potencial de contagio. Nota-se então que os profissionais do estado da Paraíba e os serviços de saúde devem estar alerta por conta da predominância da forma multibacilar da hanseníase, por ser a forma considerada incapacitante.

Ao analisar a tabela 02, é possível perceber uma grande e maior parte da alta registrada com a cura da doença, pouco número de óbitos, porém muitas fichas sem preenchimento.

Tabela 02. Número de pacientes com hanseníase de acordo com o tipo de saída.

\begin{tabular}{lccccc}
\hline Ano & Cura & Transferido Pra Outro Município & Óbito & Abanono & Não Preenchido \\
\hline 2010 & 108 & 16 & 3 & 11 & 549 \\
2011 & 679 & 31 & 12 & 30 & 82 \\
2012 & 633 & 32 & 10 & 36 & 87 \\
2013 & 443 & 24 & 11 & 17 & 239 \\
2014 & 51 & 16 & 2 & 3 & 512 \\
\hline
\end{tabular}

Diante dos resultados encontrados no período do estudo, é possível identificar que as maiorias dos pacientes saíram curado, fato justificado pela eficácia da Poliquimioterapia associada ao acompanhamento multidisciplinar na prevenção e tratamento de incapacidades. $\mathrm{O}$ tratamento do paciente com hanseníase é fundamental para cura e também para fechar a fonte de infeção, interferindo na cadeia de transmissão da doença (PEREIRA JUNIOR, 2011) Observamos uma queda nesses números no ano de 2014 associadas com um aumento de fichas não preenchidas, baseada na falha da equipe de profissionais insuficientes. $\mathrm{O}$ número de óbito apresentado foi muito pequeno em relação à cura, indicando o baixo índice de mortalidade da doença. A taxa de abandono do tratamento vem sendo amenizada ao passar dos anos. O ano de 2011 houve o maior número de cura da hanseníase, pois foi o ano em que foram notificados os maiores índices da doença.

\section{CONCLUSÃO}

Existe uma diminuição lenta nos casos de hanseníase entre 2010 e 2014 no Estado da Paraíba;

Ocorre alta endemia da forma tardia da doença, grandes números de casos da forma multibacilar indicando uma certa precariedade no atendimento realizado pelos serviços de saúde.

\section{REFERÊNCIAS}

ARAÚJO, M.G. Hanseníase no Brasil. Rev. Soc. Bras. Med. Trop. n. 3, v.36, p. 373- 382, 2003.

BRITO, K. K. G; ARAÚJO, D. A. L; UCHÔA, M. N. Epidemiologia da hanseníase em um estado. Rev. Enferm. UFPE online. v.8, p. 2686-2693. 2014

GOMES, R; NASCIMENTO, E. F; ARAúJO, F. C. Por que os homens buscam menos os serviços de saúde do que as mulheres? As explicações de homens com baixa escolaridade e com ensino superior. Cod. Saúd. Pública. n. 3, v. 23, p. 565-574, 2007.

IMBIRIBA, E. B; BASTA, P. C; PEREIRA, E. S.; LEVINO, A.; GARNELO, L. Hanseníase em populações indígenas do Amazonas, Brasil: um estudo epidemiológico nos municípios de Autazes, Eirunepé e São Gabriel da Cachoeira (2000 a 2005). Cad. Saúd. Pública. n. 5, v. 25, 2009.
LIMA, H. M. N; SAUAIA, N; COSTA, V. R. L; NETO, G. T. C; FIGUEIREDO, P. M. S. Perfil epidemiológico dos pacientes com hanseníase atendidos em Centros de Saúde em São Luís, MA. Rev. Bras. Clin. Med. n.8, v.4, p.323-327, 2010.

MAGALHÃES, M. da C. C.; ROJAS, L. I. Diferenciação territorial da hanseníase no Brasil. Epidemiol. Serv. Saúde, Brasília , v. 16, n. 2, p. 75-84, jun. 2007.

MELÃO, S.; BLANCO, L. O; MOUNZER, N.; VERONEZI, C. C. D.; SIMÕES, P. W. T. A. Perfil epidemiológico dos pacientes com hanseníase no extremo sul de Santa Catarina, no período de 2001 a 2007. Rev. Soc. Bras. Med. Trop. n. 44, v. 1, p. 79-84, 2011.

PEREIRA JUNIOR, F. A. C. Motivos do abandono ou interrupção do tratamento da hanseníase: uma revisão sistemática da literatura. 2011. Monografia (Gestão de saúde coletiva)- Fundação Oswaldo Cruz. 2011.

SIMPSON, C. A.; FONSÊCA, L. C. T; SANTOS, V. R. C. Perfil do doente de hanseníase no estado da Paraíba. Ver. Hansen. Int. v.35, p. 33-40, 2010.

SOUSA, B. R. M; MORAES, F. H.; ANDRADE, J. S.; LOBO, E. S.; MACEDO, E. A.; PIRES, C. A. A.; DAXBACHER, E. L. R. Educação em saúde e busca ativa de casos de hanseníase em uma escola pública em Ananindeua, Pará, Brasil. Rev. Bras. Med. Farm. Comunidade. n. 8, v.27, p. 143-149,2013.

SOUSA, V. M. Situação epidemiológica da hanseníase no município de Campina Grande- PB, 2005 a 2009. 2012. Monografia (Enfermagem)- Universidade Estadual da Paraíba. 2012.

SOUZA, C.S. Hanseníase: formas clínicas e diagnóstico diferencial. Medicina, Ribeirão preto. Simpósio: Hanseníase. n.30, p.325-334,1997.

XIMENES NETO, F. R. G.; LIBERATO, B. T. G.; MARTINS, F. R.; MARTINS, A. F.; CARVALHO FILHO, J. P.; SILVA, M. G. C. Epidemiologia da hanseníase no município de Cariré- Ceará, 2001 a 2010. Rev. Elet. Ges. Saúde. n. 3, v. 4, p. 829-842, 2010. 